\title{
IMPRO: IMMERSIVE PROTOTYPING IN VIRTUAL ENVIRONMENTS FOR INDUSTRIAL DESIGNERS
}

\author{
S. Stadler ${ }^{1, \bigotimes}$, H. Cornet $^{1}$, D. Mazeas ${ }^{1}$, J.-R. Chardonnet ${ }^{2}$ and F. Frenkler ${ }^{3}$ \\ ${ }^{1}$ TUMCREATE Ltd, Singapore, ${ }^{2}$ Arts et Métiers ParisTech, France, ${ }^{3}$ Technical University of Munich, Germany \\ $\triangle$ sebastian.stadler@tum-create.edu.sg
}

\begin{abstract}
Computer-Aided Design (CAD) constitutes an important tool for industrial designers. Similarly, Virtual Reality (VR) has the capability to revolutionize how designers work with its increased sense of scale and perspective. However, existing VR CAD applications are limited in terms of functionality and intuitive control. Based on a comparison of VR CAD applications, ImPro, a new application for immersive prototyping for industrial designers was developed. The user evaluations and comparisons show that ImPro offers increased usability, functionality, and suitability for industrial designers.
\end{abstract}

Keywords: immersive prototyping, industrial design, virtual reality (VR), human-computer interaction, computer-aided design (CAD)

\section{Introduction}

The design process describes the approach, strategy, steps, and activities industrial designers undergo to develop a product, service, system, or experience. During the Design phase, two-dimensional sketches are usually created and transformed into 3D models in a tangible way as a basis for design reviews and decision-making. Several tools can be used for this: pencil and paper for technical drawings, physical prototyping, CAD, and recently Virtual Reality (VR). The introduction of CAD revolutionized the way industrial designers work since it led to improved modelling capabilities and increased efficiency in terms of accuracy and product quality. Twenty years ago, the design community already foresaw that traditional methods like sketching and drawing of concepts would be enhanced by methods that utilize VR (Cross, 1999). Our hypothesis is that the introduction of VR into CAD prototyping during the Design phase can enhance the capabilities and way of working for industrial designers since it could lead to faster decision-making and product development due to advantages such as increased sense of scale and enhanced perspectives for immersive prototyping. A variety of commercial applications already exist on the market for the creation of 3D models in virtual environments. However, to the best of our knowledge, appropriate applications in terms of usability and suitability especially for industrial designers for 3D modelling in VR are still missing. Therefore, the overall goal of the present study is to develop and evaluate a new application for immersive prototyping in virtual environments (ImPro) that offers usability and functionality to make it suitable specifically for industrial designers.

\section{Related work}

VR helps to design, develop, and evaluate concepts before creating high-cost physical prototypes (Berg and Vance, 2016). The majority of design methods involving VR are utilized in later phases of 
the design process for evaluating purposes. These evaluations can be conducted for instance via usability tests, user observations, ergonomic studies, virtual assembly simulations, or immersive visualizations (Berg and Vance, 2016; Boothroyd, 1994; Mihelj et al., 2013; Ottosson, 2002; Rieuf and Bouchard, 2017; Stadler et al., 2019). Advantages of using these methods are an improved understanding of scale (Keeley, 2018), time and cost effectiveness (Berg and Vance, 2016; Deb et al., 2017; Wendrich, 2010), an enriched emotional component (Rieuf and Bouchard, 2017; Wendrich, 2010), increased concept quality (Akca, 2017; Boothroyd, 1994; Tovey, 1989), and improved design understanding and decision-making (Freeman et al., 2017). However, the usage of VR in the later phases of the design process also revealed limitations such as technical drawbacks such as restricted field of view (Berg and Vance, 2016), limited immersion (Stadler et al., 2019), lack of accuracy (Arora et al., 2017), and absence of haptic feedback (Bishop et al., 2001). Rieuf and Bouchard (2017) state that VR is scarcely used in early phases of the design process. However, technological advances in VR such as reduction in costs and improved Human-Computer Interaction methodologies offer new opportunities to integrate VR in industrial design application.

For the development of concepts in the Design phase, sketches are transformed into 3D models with the help of CAD applications (Heufler, 2004; Hirsch, 2014). The implementation of CAD into the Design phase revolutionized the way industrial designers create products since it led to improved modelling capabilities, simplification, and increased efficiency in terms of accuracy and quality of products (Akca, 2017; Boothroyd, 1994; Cross, 2006; Tovey, 1989). Industrial designers usually use desktop-based CAD applications such as Rhinoceros (2019) and 3ds Max (Autodesk, 2019). Commercial VR applications for CAD are already available on the market, such as Google Blocks (Google, 2017), Microsoft Maquette (Microsoft, 2019), Mindesk (Mindesk, 2019), Gravity Sketch (Gravity Sketch, 2017), and flyingshapes (Flyingshapes, 2019). However, the extent of VR's impact on CAD development in terms of time and quality of outcome has yet to be clarified. Furthermore, the tools of these commercial VR applications for creating and transforming volumes and surfaces as well as the user interface and navigation vary from application to application. Additionally, usability and functionality of these applications and thus, their suitability specifically for industrial designers has yet to be investigated. In the present paper, a selection of three commercial VR applications is considered, namely Google Blocks, Microsoft Maquette, and Mindesk. Furthermore, a selection of commercial desktopbased CAD applications is compared with VR CAD applications in terms of time and quality of outcome.

An interface or product is considered as usable when the user 'can do what he or she wants to do the way he or she expects to be able to do it, without hindrance, hesitation, or questions' (Rubin and Chisnell, 2008). Usability can be evaluated with the help of methods like usability testing (Rubin and Chisnell, 2008), cognitive walkthrough (Polson et al., 1992), heuristic evaluation (Nielsen and Molich, 1990), or the System Usability Scale (SUS) (Brooke, 1996).

Functionality is defined as the range of functions that an application can perform and the quality of being useful, practical and right for the intended purpose (Cambridge Dictionary, 2014). In the present study, functionality is defined as the availability and ease of using the tools (e.g. for creating a primitive) that are provided in the respective application.

Out of the aforementioned considerations, the following problem statements were defined:

- The functionalities of existing VR CAD applications are insufficient for industrial designers

- The usability of existing commercial VR applications for industrial designers is not ensured

- The impact of using VR for CAD on the model quality and time needed is unknown

Therefore, the objective of the present study is to develop a VR application for immersive prototyping with a focus on its suitability for industrial designers (in terms of usability and functionality) as well as sense of scale, perspective, time efficiency and intuitive interaction. Beyond the evaluation in terms of usability, functionality, and thus suitability for industrial designers, it will be investigated how VR impacts the time consumption, and quality of CAD models compared to desktop-based CAD applications. The contribution of the newly developed VR application lies in its usability and functionality that are derived from an analysis of commercially available VR CAD applications. This analysis was conducted to obtain a set of guidelines as a basis for the development of the application. 


\section{Methods}

The following methods have been applied in order to identify the required functionality of ImPro as well as evaluating its usability and the quality of outcome by comparing it with commercially available CAD applications both in VR and using desktop-based applications (Figure 1).

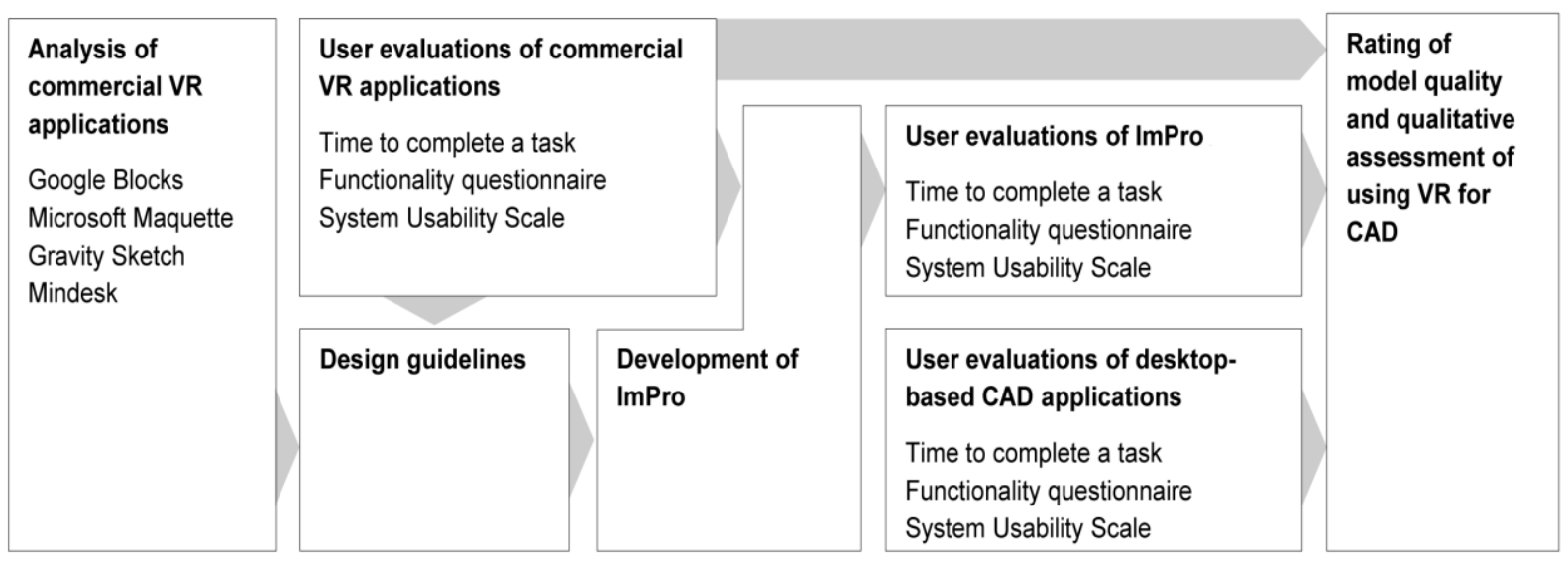

Figure 1. Overview of methods used for developing and evaluating ImPro

In the beginning, an analysis of commercial VR CAD applications was conducted for deriving similarities in functionality for creating and transforming objects as well as scene navigation. Thus, a basic functionality was identified for the implementation into ImPro such as the creation of a box or sphere, rotating objects, and navigation through teleportation inside the scene. The functionality was identified by comparing the available tools (and tool categories) per VR CAD application. Therefore, if a tool (e.g., the creation of a box) was available in all three commercial VR CAD applications, it was identified as "necessary". If a tool was available in two of the three applications, it was labelled as "advantageous". Lastly, if a tool was only present in one of the three commercial applications, it was considered as "not essential". Based on this procedure, the functionality for ImPro was derived.

Subsequently, 14 participants ( 4 female, 10 male) with an age range of 21 to 31 years ( $M=26.3$, S.D. $=3.44$ ) were asked to complete a task by using the commercially available CAD applications in VR. All participants worked in academic environment as research assistants andwere experts in using desktop-based CAD applications and thus, were considered as lead users. Preceding the test, the participants were introduced to the procedure and a consent agreement was signed. In order to minimize the risk of distorted results due to the test sequence, the CAD applications were tested in randomized order. Before starting the test for each commercial VR application, each participant had time to familiarize themselves with the virtual environment and input controls. During the test, the participants had the task to rebuild a low-complexity 3D model that was shown to them without any influence or help from the experimenters by using one of the aforementioned CAD applications and its tools. The object solely consisted of primitives that were arranged in a specific way. All chosen 3D models had a similar creation complexity (i.e., using similar tools and requiring the same amount of primitives to create the model) to ensure comparability. One specific 3D model was assigned to one commercial application (an overview of 3D models is displayed in Table 1). We collected the time each participant needed to complete the task for creating the respective 3D model. The HTC Vive Pro was used with two HTC Vive Pro Controllers in combination with a high performance computer in a six degree of freedom setup (i.e. an empty meeting room with an approximately $2.5 \mathrm{~m}^{2}$ area that was tracked by two diagonally positioned HTC Lighthouse 2 trackers).

Following the user evaluation of the commercial VR CAD applications, a functionality questionnaire and the SUS were filled out by each participant. Responses for the questionnaires were rated upon a five-point Likert scale. The functionality questionnaire consisted of ten questions that were formulated to derive the ease of using the tested application: 
Q1: Navigating in the menu was not a problem for me

Q2: Navigating in the scene was not a problem for me

Q3: Changing the scale of the scene was not a problem for me

Q4: Finding the features I was looking for was not a problem for me

Q5: Creating a 3D primitive was not a problem for me
Q6: Drawing in 3D was not a problem for me

Q7: Deleting an object was not a problem for me

Q8: Modifying an object was not a problem for me

Q9: Duplicating an object was not a problem for me

Q10: Undoing and redoing the last action was not a problem for me

The results from the user evaluation including the functionality questionnaire, the SUS, and time to complete the task gave insights into the usability and functionality of each commercial VR CAD application. Especially aspects that were identified as more usable in one commercial application than in another commercial application were considered for the development of ImPro (e.g., if the functionality questionnaire indicated that users find the user interface of Google Blocks more intuitive than Microsoft Maquette, this was considered in the development of ImPro).

In parallel to the first user evaluation, the development of ImPro started based on the findings of the analysis of commercial applications. Additionally, the insights from the first user evaluations were incorporated into the development.

Subsequently, ImPro and two desktop-based CAD applications (i.e., Rhinoceros 3D and 3ds Max) were tested following the same procedure as the first user evaluation. A desktop-computer with the same hardware specifications was used for the tests involving the desktop-based CAD applications. A Microsoft keyboard and mouse were used as input devices. The comparison of the desktop-based applications with the VR CAD applications showed how the usage of VR impacts CAD development in terms of development time, usability, functionality and model quality. Table 1 shows a summary of the low-complexity models that each participant had to create by using a specific application.

Table 1. Overview of the 3D models that had to be created by the participants

\begin{tabular}{|c|c|c|c|c|c|c|}
\hline Category & \multicolumn{4}{|c|}{ VR-based } & \multicolumn{2}{c|}{ Desktop-based } \\
\hline Application & Blocks & Maquette & Mindesk & ImPro & Rhinoceros & 3ds Max \\
\hline 3D Model & & & & & & \\
\hline
\end{tabular}

As a last step, the model quality of each application was assessed by three researchers from the same department with a background in industrial design and psychology. The evaluators compared the created 3D models with the reference models and rated them based on four categories: i) correct amount of primitives, ii) correct shapes of primitives, iii) correct placement and orientation of primitives to create the model, and iv), correct proportions of the primitives and 3D model. For each category, up to five points were awarded by the evaluators. The average score of all totalled categories showed the respective model's quality. Finally, after the user evaluations, a qualitative assessment was conducted in which participants were asked open-ended questions about their subjective perception regarding the potential of using VR for CAD.

\section{Results}

\subsection{Analysis of commercial VR applications}

Figure 2 shows interfaces of all three commercial VR applications that were part of the investigation. 

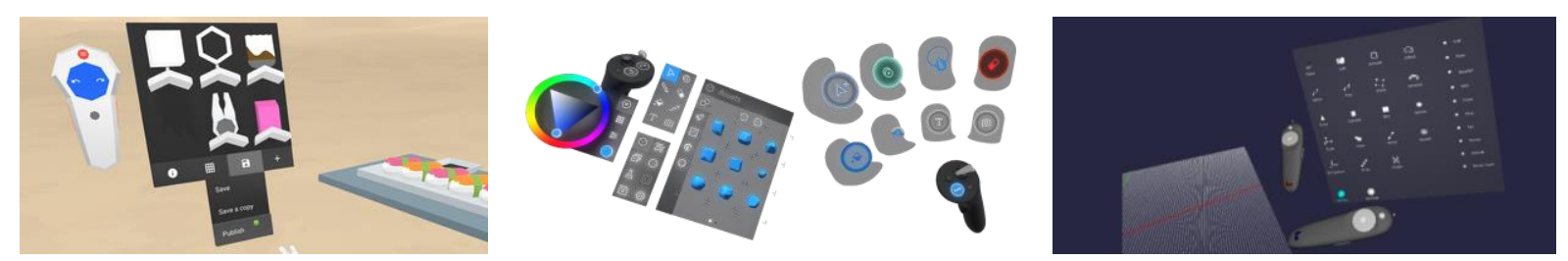

Figure 2. Interfaces of commercial applications (left: Google Blocks; middle: Microsoft Maquette; right: Mindesk)

Table 2 summarizes the analysis of the commercial VR applications regarding their functionality.

Table 2. Comparison of tools of commercial applications

\begin{tabular}{|l|l|l|l|}
\hline Application/Function & Blocks & Maquette & Mindesk \\
\hline Primitive & $\begin{array}{l}\text { Cone, Cube, Cylinder, } \\
\text { Sphere, Torus }\end{array}$ & $\begin{array}{l}\text { Box, Cone, Cylinder, } \\
\text { Sphere }\end{array}$ & $\begin{array}{l}\text { Box, Cone, Cylinder, } \\
\text { Sphere, +40 shapes }\end{array}$ \\
\hline Transform & $\begin{array}{l}\text { Select, Scale, Copy, } \\
\text { Group, Erase, Change } \\
\text { colour }\end{array}$ & $\begin{array}{l}\text { Scale, Copy, Move, } \\
\text { Rotate, Erase, Change } \\
\text { colour }\end{array}$ & $\begin{array}{l}\text { Scale, Move, Rotate, } \\
\text { Erase, Group }\end{array}$ \\
\hline Navigation & Grip buttons & Grip buttons & Grip buttons, teleport \\
\hline
\end{tabular}

Based on the comparison, the following tools were identified as essential for ImPro:

- Basic object creation tools for primitives, including, box, cone, cylinder, and sphere

- Basic transformation tools like select, move, scale, copy, group, erase

- Navigation tools for moving inside the virtual environment and scaling the scene

\subsection{Design guidelines and the development of ImPro}

Based on the analysis of commercial VR applications and the user evaluations of commercial VR applications, the following design guidelines were derived for the development of ImPro:

- Availability of the creation and modification tools for primitives

- Availability of navigation tools

- Display all available tools in a one-layered user-interface

- Possibility to hide the tools to increase the visibility of the scene and 3D models

- Use intuitive tool icons

- Show the user which tool is selected

- Make the undo and redo buttons accessible und usable at any time

- Include visual guidelines (i.e. pointers in front of the input devices) to increase accuracy

Figure 3 shows the user interface (left) as well as tools such as the undo button (middle) and a visual support to identify the selected tools (right).
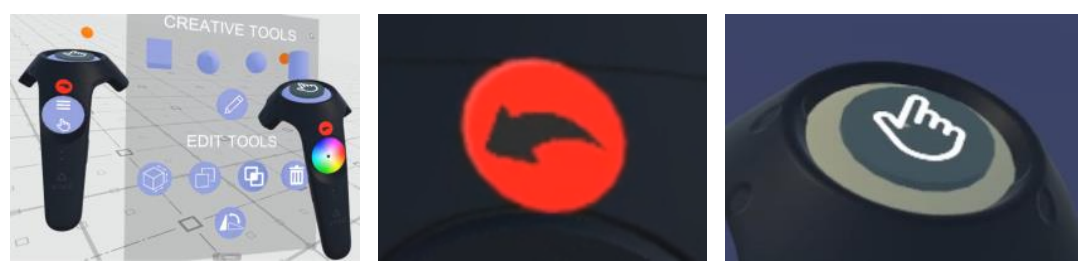

Figure 3. User interface and tools of ImPro

Additional features were added based on descriptive statements from the test participants after conducting the user evaluation such as the creation of a primitive with a locked aspect ratio by pressing a button and visual guidelines to increase the precision when creating and modifying primitives (i.e., visual guides in front of the input). 


\subsection{User evaluations of VR- and desktop-based CAD applications and ImPro}

The results of the user evaluations of commercial VR applications, ImPro, and desktop-based CAD applications are combined for clarity and comparability, even though it does not follow the chronological procedure of evaluations pictured in Figure 1. Table 3 shows a selection of 3D models that were created by the participants while using the different VR- and desktop-based CAD applications and ImPro. The models' colour schemes were not part of the evaluation.

Table 3. Selection of models created by the participants

\begin{tabular}{|c|c|c|c|c|c|c|c|}
\hline Model/Application & Model 1 & Model 2 & Model 3 & Model 4 & Model 5 & Model 6 & Model 7 \\
\hline Blocks & & & & & & & \\
\hline ImPro & & & & & & & \\
\hline Rhinoceros & & & & & & & \\
\hline
\end{tabular}

\subsubsection{Time to complete the task}

Table 4 shows the average time the participants needed to complete the task per application.

Table 4. Average times to complete the task per application

\begin{tabular}{|c|c|c|}
\hline Category & Application & Average time in sec \\
\hline \multirow{4}{*}{ VR-based } & Blocks & 430 \\
\cline { 2 - 3 } & Maquette & 832 \\
\cline { 2 - 3 } & Mindesk & 524 \\
\cline { 2 - 3 } & ImPro & $\mathbf{2 3 0}$ \\
\hline \multirow{2}{*}{ Desktop-based } & Rhinoceros & 456 \\
\cline { 2 - 3 } & 3 ds Max & 508 \\
\hline
\end{tabular}

Table 4 shows that the participants needed a similar amount of time for completing the task with Google Blocks, Mindesk, Rhinoceros 3D, and 3ds Max. This indicates that VR did not influence the time to complete the task. Furthermore, the participants needed considerably more time to complete the task by using Microsoft Maquette. By using ImPro, the participants needed less time than with any other application.

\subsubsection{Functionality questionnaire}

Table 5 shows the average scores of the functionality questionnaire considering tools like the creation and transformation of primitives and scene navigation.

Table 5. Average functionality scores

\begin{tabular}{|c|c|c|}
\hline Category & Application & Average functionality score \\
\hline \multirow{3}{*}{ VR-based } & Blocks & 4.3 \\
\cline { 2 - 3 } & Maquette & 3.6 \\
\cline { 2 - 3 } & Mindesk & 3.7 \\
\cline { 2 - 3 } & ImPro & $\mathbf{4 . 4}$ \\
\hline \multirow{2}{*}{ Desktop-based } & Rhinoceros & 4.3 \\
\cline { 2 - 3 } & 3ds Max & 4.3 \\
\hline
\end{tabular}


Table 5 shows that Google Blocks and ImPro are comparable with the desktop-based CAD applications in terms of functionality, while Microsoft Maquette and Mindesk achieved lower ratings.

In addition to the functionality questionnaire, the participants gave the following statements for the different applications:

- The large amount of tools and its clustering in the user interface of Maquette was confusing.

- Deleting an object was the easiest in Google Blocks since there was an icon of an eraser and once the eraser was selected the input device changed its shape accordingly.

- Even though there are more tools available in Mindesk than Google Blocks, the tools in Mindesk remain easy to find since the interface panel shows all tools at once. Additionally, when the interface of Mindesk is not needed, it can be hidden.

\subsubsection{System Usability Scale}

Table 6 shows the average SUS scores for the respective commercial application.

Table 6. Average SUS scores

\begin{tabular}{|c|c|c|}
\hline Category & Application & Average SUS score \\
\hline \multirow{3}{*}{ VR-based } & Blocks & 75.5 \\
\cline { 2 - 3 } & Maquette & 44.2 \\
\cline { 2 - 3 } & Mindesk & 62.5 \\
\cline { 2 - 3 } & ImProVE & $\mathbf{7 7 . 3}$ \\
\hline \multirow{2}{*}{ Desktop-based } & Rhinoceros & 68.4 \\
\cline { 2 - 3 } & 3ds Max & 55.4 \\
\hline
\end{tabular}

Following the proposed data analysis for the SUS, all answers from the participants were converted into a score that reaches from 0 to 100 whereas a score of 100 represents the best possible usability. A usability score of 68 and above is considered as above average (Brooke, 1996). Google Blocks and ImPro had the best SUS scores. Rhinoceros 3D also achieved a usability score that is barely considered as "above average". Following the SUS scoring, all other applications are considered as "below average" in terms of usability.

\subsubsection{Quality of model}

Table 7 shows the average scores of model quality for each application as rated by the evaluators.

Table 7. Average scores for the quality of model

\begin{tabular}{|c|c|c|c|c|c|c|}
\hline Category & Application & $\begin{array}{c}\text { Amount of } \\
\text { primitives }\end{array}$ & $\begin{array}{c}\text { Correct tools } \\
\text { for object } \\
\text { creation }\end{array}$ & $\begin{array}{c}\text { Correct } \\
\text { assembly and } \\
\text { orientation }\end{array}$ & $\begin{array}{c}\text { Correct } \\
\text { proportions }\end{array}$ & $\begin{array}{c}\text { Average } \\
\text { scores }\end{array}$ \\
\hline \multirow{4}{*}{ VR-based } & Blocks & 4.79 & 4.86 & 3.57 & 4.00 & 4.30 \\
\cline { 2 - 7 } & Maquette & 4.57 & 4.57 & 4.07 & 3.36 & 4.14 \\
\cline { 2 - 7 } & Mindesk & 4.79 & 4.64 & 3.86 & 3.43 & 4.18 \\
\cline { 2 - 7 } & ImPro & $\mathbf{5 . 0 0}$ & $\mathbf{4 . 9 3}$ & $\mathbf{3 . 8 2}$ & $\mathbf{3 . 6 4}$ & $\mathbf{4 . 3 5}$ \\
\hline \multirow{2}{*}{$\begin{array}{c}\text { Desktop- } \\
\text { based }\end{array}$} & Rhinoceros & 5.00 & 4.79 & 4.21 & 2.79 & 4.20 \\
\cline { 2 - 7 } & 3 ds Max & 5.00 & 4.93 & 4.00 & 3.00 & 4.23 \\
\hline
\end{tabular}

Table 7 shows that the total average scores of each tested application lies between 4.14 and 4.35 points (out of 5 points). The table indicates that it was easier to correctly assemble the model with desktopbased CAD applications. In contrast, the proportion scores imply that the models' proportions were better by using VR than desktop-based CAD applications. When comparing the overall average scores of the VR applications (4.24) to the desktop-based applications (4.21), it becomes visible that there are only minor differences in terms of model quality. 


\subsubsection{Qualitative assessment}

In the qualitative assessment, the participants stated that VR might improve creativity while creating 3D models as well as intuitive interaction. Furthermore, participants believe that the usage of VR in the Design phase can accelerate the development time and decision time in general. Overall, it was also mentioned that immersive prototyping in VR was very exciting and made the creation of 3D models very tangible. Nevertheless, some participants pointed out a lack of accuracy in VR CAD applications, which implies that the usage of VR is not suitable yet to generate highly detailed 3D models.

\section{Discussion}

We developed an application that offers the essential tools for developing low-complexity 3D models in an immersive environment. We focused on the factors of usability and functionality which are fundamental for industrial designers for the development of 3D models. Our contribution is fourfold. Firstly, the development and evaluation of ImPro has the potential to be used by industrial designers during the product development and especially in the Design phase with advantages over other VR applications and desktop-based applications such as increased usability and functionality. Furthermore, the quality of produced 3D models was improved when participants used ImPro. This could be particularly advantageous for the product development since it shows that VR offers even increased capabilities of producing 3D models for decision making in design reviews (e.g. for discussing product details in early stages of CAD development). A further contribution is the analysis of VR-based CAD applications in terms of functionality and usability. This analysis helps to identify aspects such as visibility of tools and clear user interface that increases and decreases the usability of each respective commercial application which led to the design guidelines of developing ImPro. We anticipate that due to this comparison and the development of the design guidelines, a basic framework was built to support further research on VR CAD applications as well as facilitating potential transferability to other application fields. Lastly, the general comparison of desktop-based CAD applications and VR CAD applications showed the impact of VR on the time required to develop a low-complexity model, the functionality and usability, as well as the quality of models. This comparison showed that VR has a great potential to improve the product development.

The user evaluations and the qualitative assessment showed that the participants were excited to use the technology of VR for prototyping. This reflects the findings of Rieuf and Bouchard (2017) who concluded that the usage of VR in early phases of design can enhance the emotional component of the activity and lead to higher general engagement of the designer while completing the task. Furthermore, the participants gave positive comments during and after the test with regards to the scale of the 3D models, the possibility to look at the model from any perspective, and the intuitive and unique interaction to create 3D models. This was reflected in the model quality which showed that the proportions and the assembly of the created models were better while using VR applications. This is consistent with findings of Keeley (2018) who conducted an experiment for sketching in VR. The results highlighted a greater sense of scale and perspective from participants' side when using VR. One specific advantage of ImPro is the clear interface for selecting tools for the creation and transformation of primitives and shapes. Similar to Mindesk, it gives a clear overview of all available tools. Adapted from Google Blocks, ImPro offers visual feedback of the selected tool via icons, as well as an undo and redo button that is available at any time.

Even though visual guides were implemented, the lack of accuracy was still the biggest limitation for using VR for immersive prototyping in the conducted study. Independent of the VR application, the majority of participants stated that, compared to desktop-based CAD applications, immersive prototyping did not offer a high level of accuracy, which could potentially influence a model's quality. Furthermore, due to the simplicity of the task, we only can derive implications of VR's potential for design activities. Additional evaluations including more complex tasks are required. Arora et al. (2017) concluded that the lack of precision and accuracy is a key limitation of using VR. Furthermore, ImPro was tested after testing the commercial applications. It is anticipated that there was a learning curve for using CAD in an immersive environment which led to accelerated task completion time and positive responses during the SUS and functionality questionnaires. 
As a next step, the functionality of ImPro will be enhanced to include sketching tools and spline creation. Since this allows a seamless transformation from sketches into 3D models, it is anticipated that this functionality will improve the suitability for industrial designers. Additional research will be conducted to further improve accuracy for immersive prototyping.

\section{Conclusion}

The integration of Virtual Reality into the design process offers a range of advantages such as saving time and cost, enhanced visualizations and evaluation before building physical prototypes, as well as an intuitive interaction. The present study investigated and tested a range of commercial VR applications for CAD in immersive environments as a basis for the development of ImPro, a VR CAD application with a focus on the suitability (in terms of usability and functionality) for industrial designers. The user evaluations and comparisons of the newly developed application with existing VR- and desktop-based applications showed improved usability, functionality, increased sense of scale and enhanced perspective when using the developed application. The participants also expressed their excitement to create 3D models in virtual environments. Further research will be conducted to improve the accuracy in VR for allowing the designers to create high quality 3D models in virtual environments.

\section{Acknowledgements}

This study is part of a research programme, TUMCREATE Phase 2, funded by the National Research Foundation (NRF) Singapore under its Campus for Research Excellence and Technological Enterprise (CREATE) programme.

\section{References}

Akca, E. (2017), "Development of Computer-Aided Industrial Design Technology", Periodicals of Engineering and Natural Sciences (PEN), Vol. 5 No. 2, pp. 124-127. https://doi.org/10.21533/pen.v5i2.86

Arora, R. et al. (2017), "Experimental Evaluation of Sketching on Surfaces in VR", Proceedings of the 2017 CHI Conference on Human Factors in Computing Systems - CHI '17, Association for Computing Machinery (ACM), Denver. https://doi.org/10.1145/3025453.3025474

Autodesk (2019), 3ds max - 3d modeling and rendering software for design visualization, games, and animation, [online] Available at: https://www.autodesk.com/products/3ds-max/overview (accessed 7.10.2019).

Berg, L.P. and Vance, J.M. (2016), "Industry use of virtual reality in product design and manufacturing: a survey", Virtual Reality, Vol. 21 No. 1. https://doi.org/10.1007/s10055-016-0293-9

Bishop, I.D., Wherrett, J.A.R. and Miller, D.R. (2001), “Assessment of path choices on a country walk using a virtual environment”, Landscape and Urban Planning, Vol. 52 No. 4, pp. 225-237. https://doi.org/10.1016/ S0169-2046(00)00118-3

Boothroyd, G. (1994), "Product design for manufacture and assembly", Computer-Aided Design, Vol. 26 No. 7 , pp. 505-520. https://doi.org/10.1016/0010-4485(94)90082-5

Brooke, J. (1996), "SUS: A “quick and dirty" usability scale”, In: Jordan, P.W., Thomas, B., Weerdmeester, B. and McClelland, A.L. (Eds.), Usability Evaluation in Industry, Taylor \& Francis, London.

Cambridge Dictionary (2014), Virtual Reality, [online] Cambridge University Press, Available at: https://dictionary. cambridge.org/de/worterbuch/englisch/virtual-reality?q=Virtual+Reality (accessed 26.01.2019).

Cross, N. (2006), Designerly ways of knowing, Designerly Ways of Knowing, Springer, London. https://doi.org/ $10.1007 / 1-84628-301-9$

Cross, N. (1999), "Design Research: A Disciplined Conversation”, Design Issues, Vol. 15 No. 2, pp. 5-10.

Deb, S. et al. (2017), "Efficacy of virtual reality in pedestrian safety research", Applied Ergonomics, Vol. 65, pp. 449-460. https://doi.org/10.1016/j.apergo.2017.03.007

Flyingshapes (2019), Flyingshapes, [online] Available at: https://www.flyingshapes.com/ (accessed 29.08.2019).

Freeman, I.J., Salmon, J.L. and Coburn, J.Q. (2017), "CAD Integration in Virtual Reality Design Reviews for Improved Engineering Model Interaction", Proceedings of the ASME 2016 International Mechanical Engineering Congress and Exposition, ASME, Phoenix. https://doi.org/10.1115/imece2016-66948

Google (2017), Google Blocks, [online] Available at: https://vr.google.com/blocks/ (accessed 29.08. 2019).

Gravity Sketch (2017), Gravity Sketch, [online] Available at: https://www.gravitysketch.com/ (accessed 29.08.2019).

Heufler, G. (2004), Design Basics - From Ideas to Products, Niggli Verlag AG, Zürich. 
Hirsch, S. (2014), Gestaltung und Umbruch - Industrie Design als Mittel sozioökonomischer Wertschöpfung, Diplomica Verlag, Hamburg.

Keeley, D. (2018), The use of Virtual Reality Sketching in the conceptual stages of Product Design, [Department of Design \& Engineering], Bournemouth University.

Microsoft (2019), Maquette, [online] Available at: https://www.maquette.ms/ (accessed 29.08.2019).

Mihelj, M., Novak, D. and Beguš, S. (2013) Virtual Reality Technology and Applications, Springer Science+Business Media, Dodrecht. https://doi.org/10.1007/978-94-007-6910-6

Mindesk (2019) Mindesk. [online] Available at: https://mindeskvr.com/ (accessed 29.08. 2019).

Nielsen, J. and Molich, R. (1990), "Heuristic Evaluation of User Interfaces", Proceedings of the 1990 CHI Conference on Empowering People - CHI'90, Association for Computing Machinery (ACM), Seattle. https://doi.org/10.1016/0921-4526(93)90674-U

Ottosson, S. (2002), "Virtual reality in the product development process", Journal of Engineering Design, Vol. 13 No. 2, pp. 159-172. https://doi.org/10.1080/09544820210129823

Polson, P.G. et al. (1992), "Cognitive walkthroughs: a method for theory-based evaluation of user interfaces", International Journal of Man-Machine Studies, Vol. 36 No. 5, pp. 741-773. https://doi.org/10.1016/00207373(92)90039-N

Rhinoceros (2019), Rhinoceros, [online] Available at: https://www.rhino3d.com/ (accessed 7.10.2019).

Rieuf, V. and Bouchard, C. (2017), "Emotional activity in early immersive design: Sketches and moodboards in virtual reality", Design Studies, Vol. 48, pp. 43-75. https://doi.org/10.1016/j.destud.2016.11.001

Rubin, J. and Chisnell, D. (2008), Handbook of usability testing: How to plan, design, and conduct effective tests, Wiley Publishing, Indianapolis.

Stadler, S., Cornet, H. and Frenkler, F. (2019), “A Study in Virtual Reality on (Non -) Gamers, Attitudes and Behaviors", Proceedings of the 26th IEEE Conference on Virtual Reality and 3D User Interfaces, IEEE, Osaka. https://doi.org/10.1109/VR.2019.8797750

Stadler, S. et al. (2019), "A Tool, not a Toy: Using Virtual Reality to Evaluate the Communication Between Autonomous Vehicles and Pedestrians", In: tom Dieck, M.C. and Jung, T.H. (Eds.), Augmented Reality and Virtual Reality, Springer Nature Switzerland, Cham.

Tovey, M. (1989), "Drawing and CAD in industrial design”, Design Studies, Vol. 10 No. 1, pp. 24-39. https://doi.org/10.1016/0142-694X(89)90022-7

Wendrich, R.E. (2010), "Raw shaping form finding: Tacit tangible CAD", Computer-Aided Design and Applications, Vol. 7 No. 4, pp. 505-531. https://doi.org/10.3722/cadaps.2010.505-531 